\title{
Delays in the management of lung cancer
}

\author{
P J M George
}

Surgery remains the treatment of choice for patients with early stage lung cancers. ${ }^{1}$ The aim is to remove all malignant tissue before local invasion and/or distant metastatic spread has occurred. Current practices are therefore directed towards diagnosing and staging lung cancers as early and efficiently as possible, while also ensuring that patients with operable tumours undergo surgery promptly. The Standing Medical Advisory Committee has recently recommended that the interval between a patient's first presentation to his general practitioner and resection should be no longer than $6-8$ weeks. $^{2}$

Billing and Wells recently presented data on the time taken from first presentation to surgery in a consecutive series of 38 patients with suspected or proven primary lung cancers. ${ }^{3}$ The mean interval was found to be 109 days, of which an average of 58 days was taken up with diagnostic and staging investigations. The authors expressed concern that delays of this magnitude, which are twice that regarded as acceptable, ${ }^{2}$ only serve to heighten the disruption to patients' lives and possibly jeopardise their chances of cure.

There can be little doubt that patients with suspected lung cancer suffer in many ways while undergoing diagnostic and staging investigations. This is sufficient justification alone for expediting their management. Although there is no evidence to suggest that management delays adversely affect clinical outcomes, it seems likely that delays of four months, which approximate to one tumour volume doubling time for non-small cell carcinoma, ${ }^{4}$ will ensure that some patients will require more extensive resections while others with borderline operable tumours at presentation will become inoperable. Common sense therefore dictates that we should manage our patients more efficiently.

\section{The need for change}

There is now a growing body of evidence to suggest that the management of lung cancer in the UK falls short of accepted standards of practice in several other important respects. ${ }^{5}$ Reviews of unselected groups of patients with lung cancer have recently shown wide variations in management practices which relate to the specialty interest of the hospital clinician to whom the patient has been referred ${ }^{6}$ and facilities that are available locally in the patient's hospital of origin. ${ }^{7}$

A substantial number of patients are not referred to chest physicians or consultants with an interest in thoracic oncology and there is evidence to suggest that these patients are less likely to receive active treatments such as surgery, radiotherapy, or chemotherapy. ${ }^{56}$ As the proportion of patients with lung cancer who undergo resection in the UK is substantially less than in Europe and North America, ${ }^{2}$ there must be concern that some patients with operable tumours are being denied the chance of curative surgery in our present system.

Billing and Wells ${ }^{3}$ argued the case for establishing specialist centres as proposed by the Department of Health. ${ }^{8}$ They suggest that patients should be managed intensively as inpatients in these centres with the aim of completing their diagnostic and staging investigations within 1-2 days. Such a system would have the advantage of expediting definitive treatments such as surgery while also ensuring higher and more uniform standards of management generally.

\section{Prospects of earlier treatment through earlier diagnosis}

An alternative approach to achieving earlier treatment is by diagnosing lung cancers at earlier stages in their development. Billing and Wells advocated the establishment of screening programmes. ${ }^{3}$ Trials of lung cancer screening, however, have produced rather disappointing results in the past. The most celebrated trials were conducted in the 1970 s at the Mayo Clinic, ${ }^{9}$ Johns Hopkins ${ }^{10}$ and Memorial-Sloane Kettering Hospitals. ${ }^{11}$ Each trial involved over 10000 men, aged 45 years or more, who were regarded as being at risk of developing lung cancer on the basis of their smoking histories. The trials were designed to assess the value of screening using sputum cytological testing in combination with regular chest radiography. In the trials conducted at the Johns Hopkins and Memorial Sloane-Kettering Hospitals subjects were randomised to either annual chest radiography alone (control group) or annual chest radiography with four monthly sputum cytological tests (screened group). At the Mayo Clinic the control group underwent annual chest radiography and sputum cytological tests while the screened group underwent these investigations every four months. The results were broadly similar; although more cases of early stage lung cancer were detected in the screened populations with improved five year survivals, the eventual mortality from lung cancer was identical to that of the control groups. ${ }^{12-14}$ The improved survival was therefore attributed to lead time and length time bias.

The prevailing view at the time of these trials was that chest radiography was unlikely to be an effective screening tool and so its usefulness was not evaluated. Chest radiographic screening has subsequently been evaluated in casecontrol studies ${ }^{1516}$ and has not been shown to have a significant impact on lung cancer mortality. Similarly, chest radiographic screening for lung cancer in chromate workers has failed to demonstrate a benefit of screening when the survival figures of patients compliant and non-compliant with screening who later developed lung cancer were compared. ${ }^{17}$

Despite the disappointment of these trials, interest continues in developing techniques that might allow lung cancers to be diagnosed at earlier stages when prompt intervention may improve the chances of cure. At Johns Hopkins Hospital sputum samples obtained during the screening programme were banked in an archive while follow up data were collected on the providers of these specimens. This has allowed investigators to evaluate more recently developed tumour markers using material where the eventual clinical outcome is known. ${ }^{18}$ For example, immunostaining with murine monoclonal antibodies to small cell and non-small cell antigens has shown a strong correlation between positive staining and the eventual development of lung cancer. ${ }^{19}$ Using samples obtained, on average, 20 months before the detection of cancer and from comparable controls who did not develop cancer, 
immunostaining was found to be $91 \%$ sensitive and $88 \%$ specific.

Similarly, a polymerase chain reaction based assay has demonstrated ras or $p 53$ gene mutations in sputum samples from the Johns Hopkins archive up to one year before a diagnosis of lung cancer was made. ${ }^{20}$ The study was based upon 15 patients who developed adenocarcinoma whose sputum samples were consistently negative by conventional cytological tests. Ten of these 15 patients were found to have Ras or $p 53$ gene mutations in their resected primary tumours, while the corresponding mutation was found in the sputum of eight cases.

Developments in diagnostic bronchoscopy may also facilitate the detection of early lung cancers. A novel bronchoscopic imaging system has been developed which is thought to enhance the identification of dysplasia and carcinoma in situ by exploiting differences in autofluorescence of normal and abnormal bronchial mucosa. ${ }^{21}$ When fluorescence bronchoscopy is performed in conjunction with conventional white light bronchoscopy in patients thought to be at high risk of malignancy, significantly more areas of dysplasia and carcinoma in situ have been found. ${ }^{2122} \mathrm{~A}$ large multicentre study is now being conducted in Europe to evaluate the possible advantages of this system further.

These developments raise hope that we will have the capability of diagnosing lung cancers at much earlier stages when prompt intervention is more likely to eradicate the disease. If effective screening strategies can be developed, the burden on our already stretched lung cancer service will undoubtedly be increased.

\section{Conclusions}

There can be little doubt that the management of lung cancer in the UK is often suboptimal. Muers and Haward have recently argued the case for establishing higher and more uniform standards of management. ${ }^{5}$ In addition, services for lung cancer need to be organised so that all of our patients can be assessed and treated promptly and efficiently. At the very least, this will reduce the anxiety and suspense that patients suffer and may also ensure that more patients are offered curative surgery. If we can develop effective methods for early diagnosis, prompt management may also improve the outcome of this common and lethal disease.

University College London Hospitals,

Department of Thoracic Medicine,

The Middlesex Hospital,

Mortimer Street,

London W1N $8 A A$, UK

1 Pearson FG. Current status of surgical resection of lung cancer. Chest 1984 106:337-9s

2 Standing Medical Advisory Committee. Management of lung cancer: current clinical practices. London: Department of Health, 1994.

3 Billing JS, Wells FC. Delays in the diagnosis and treatment of lung cancer. Thorax 1996;51:903-6.

4 Geddes DM. The natural history of lung cancer: a review based on rates of tumour growth. Br f Dis Chest 1979;73:117.

5 Muers MF, Haward RA. Management of lung cancer. Thorax 1996;51: $557-60$

6 Brown JS, Eraut D, Trask C, Davison AG. Age and the treatment of lung cancer. Thorax 1996;51:564-8.

7 Fergusson RJ, Gregor A, Dodds R, Kerr G. Management of lung cancer in South East Scotland. Thorax 1996;51:569-74.

8 Department of Health, Welsh Office. A policy framework for commissioning cancer services. A report of the Expert Advisory Group on Cancer to the Chief Medical Officers of England and Wales. London: Department of Health, 1995 .

9 Fontana RS, Sanderson DR, Taylor WF, Woolner LB, et al. Early lung cancer detection: results of the initial (prevalence) radiologic and cytologic screening in the Mayo Clinic study. Am Rev Respir Dis 1984;130:561-5.

10 Frost JK, Ball WC, Levin ML, Tockman MS, et al. Early lung cancer detection: results of the initial (prevalence) radiologic and cytologic screening in the Johns Hopkins study. Am Rev Respir Dis 1984;130:549-54.

11 Flehinger BJ, Melamed MR, Zaman MB, Heelan RT, et al. Early lung cancer detection: results of the initial (prevalence) radiologic and cytologic screening in the Memorial Sloane-Kettering study. Am Rev Respir Dis 1984;130:555-60.

12 Fontana RS, Sanderson DR, Woolner LB, Taylor WF, et al. Lung cancer screening: the Mayo program. F Occup Med 1986;28:746-50.

13 Melamed MR, Flehinger BJ, Zaman MB, Heelan RT, et al. Screening for early lung cancer: results of the Memorial Sloane-Kettering study in New York. Chest 1984;86:44-53.

14 Tockman MS. Survival and mortality from lung cancer in a screened population. The Johns Hopkins Study. Chest 1986;89(Suppl):324s.

15 Ebeling K, Nischan P. Screening for lung cancer: results from a case-control study. Int $\mathcal{F}$ Cancer 1987;40:141-4.

16 Berndt R, Ebeling K, Nischan P. Screening for lung cancer in the middleaged. Int 7 Cancer 1990;45:229-30.

17 Schilling CJ, Schilling JM. Chest X ray screening for lung cancer at three British chromate plants from 1985 to 1989 . Br f Ind Med 1991;48:476-9. 18 Mulshine JL, Scott F. Molecular markers in early cancer detection. New screening tools. Chest 1995;107:280-6s.

19 Tockman MS, Gupta PK, Myers JD, Frost JK, et al. Sensitive and specific monoclonal antibody recognition of human lung cancer antigen on preserved sputum cells: a new approach to early lung cancer detection. $\mathcal{F}$ Clin Oncol 1988;6:1685-93.

20 Mao L, Hruban RH, Boyle JO, Tockman M, et al. Detection of oncogene mutations in sputum precedes diagnosis of lung cancer. Cancer Res 1994 54:1634-7.

21 Lam S, Macaulay C, Hung J, LeRiche J, et al. Detection of dysplasia and carcinoma in situ with a lung imaging fluorescence endoscope device. $\mathcal{F}$ Cardiovasc Surg 1993;105:1035-40.

22 Lam S, Macaulay C, LeRiche JC, Ikeda N, Palcic B. Early localisation of bronchogenic carcinoma. Diagn Ther Endosc 1994;1:758. 